\title{
ON CONDITIONAL INCLUSION OF MATRIX METHODS
}

\section{J. MAYER-KALKSCHMIDT}

A summation method $B$ is said to be stronger than a method $A$ if the set of $A$-summable sequences is contained in the set of $B$ summable sequences. The purpose of this paper is to study inclusion relations between methods for other sets of sequences. In the first section we shall introduce the notation. In the second we prove the two main theorems, and present some applications in the third part. Finally we shall mention possible generalizations and corresponding theorems for absolute summability.

1. A sequence $\left\{s_{n}\right\}$ is summable by the triangular matrix method ${ }^{1}$ $A=\left(a_{n v}\right)$, if the sequence

$$
\sigma_{n}=\sum_{\nu=0}^{n} a_{n \nu} s_{\nu}
$$

converges for $n \rightarrow \infty$. We denote by $A^{\prime}=\left(a_{n \nu}^{\prime}\right)$ the inverse matrix of $A$, i.e. $A^{\prime}$ is the matrix such that

$$
s_{n}=\sum_{\nu=0}^{n} a_{n^{\prime} \nu} \sigma_{\nu}
$$

With $\Omega(n)$ denoting a positive monotonic function, defined for all natural numbers ${ }^{2}$ we shall let $[A, O(\Omega)]$ stand for the set of all sequences $\left\{s_{n}\right\}$ for which $\sigma_{n}=O(\Omega(n)), n \rightarrow \infty$. The symbol $[A, o(\Omega)]$ is defined in a similar way. We say, the method $C=\left(c_{n v}\right)$ satisfies the mean value condition $(\mathfrak{M})$, if to any pair of integers $n \leqq m$ there exists a third integer $n^{\prime}$ such that

$$
\left|\sum_{\nu=0}^{n} c_{m \nu} s_{\nu}\right| \leqq K\left|\sum_{\nu=0}^{n^{\prime}} c_{n^{\prime} \nu} s_{\nu}\right|, \quad n^{\prime} \leqq n \leqq m
$$

where $K$ is a constant depending only on the method $C .^{3}$ It has been shown by Jurkat and Peyerimhoff [4], that many important methods satisfy the condition $(\mathfrak{M})$, e.g. the $(C, k)$ methods for $0 \leqq k \leqq 1$, and the Riesz methods for $0 \leqq k \leqq 1 .^{4}$

Presented to the Society, January 30, 1958; received by the editors April 21, 1958 and, in revised form, July 29, 1958.

1 We shall restrict our investigations to triangular matrices.

${ }^{2} \omega(n)$ will be used with the same definition.

${ }^{3}$ See Jurkat-Peyerimhoff $[4 ; 5]$.

${ }^{4}$ See also Bosanquet [1] and Jurkat [3]. 
2. Theorem 1. Let the method $A$ satisfy the condition $(\mathfrak{M})$ and let $\Omega(n)$ be monotonic increasing. If

then

$$
S_{n}=\sum_{\nu=0}^{n} \Omega(\nu)\left|\Delta_{\nu} \frac{b_{n \nu}}{a_{n \nu}}\right|=O(1)
$$

$$
[A, O(\Omega)] \subset[B, O(1)] \text {. }
$$

Proof. We have

Therefore

$$
\begin{aligned}
\tau_{n} & =\sum_{\nu=0}^{n} b_{n \nu} s_{\nu}=\sum_{\nu=0}^{n} \frac{b_{n \nu}}{a_{n \nu}} a_{n \nu} s_{\nu} \\
& =\sum_{\nu=0}^{n} \Omega(\nu) \Delta_{\nu} \frac{b_{n \nu}}{a_{n \nu}} \frac{1}{\Omega(\nu)} \sum_{\mu=0}^{\nu} a_{n \mu} s_{\mu} .
\end{aligned}
$$

$$
\begin{aligned}
\left|\tau_{n}\right| & \leqq \sum_{\nu=0}^{n} \Omega(\nu)\left|\Delta_{\nu} \frac{b_{n \nu}}{a_{n \nu}}\right| \frac{1}{\Omega(\nu)} K\left|\sum_{\mu=0}^{n^{\prime}} a_{n^{\prime} \mu} s_{\mu}\right| \\
& =\sum_{\nu=0}^{n} \Omega(\nu)\left|\Delta_{\nu} \frac{b_{n \nu}}{a_{n \nu}}\right| \frac{\Omega\left(n^{\prime}\right)}{\Omega(\nu)} O(1), \quad n^{\prime} \leqq \nu \leqq n .
\end{aligned}
$$

Since $\Omega(n)$ is monotonic increasing, $\Omega\left(n^{\prime}\right) / \Omega(\nu)=O(1)$ and hence the proof is complete.

Remarks. (1) Apparently $S_{n}=O(\omega(n))$ implies $[A, O(\Omega)]$ $\subset[B, O(\omega)]$. Also $S_{n}=o(\omega(n))$ implies $[A, O(\Omega)] \subset[B, o(\omega)]$.

(2) For $\Omega(n)=1$ Theorem 1 reduces to the inclusion theorem of Jurkat and Peyerimhoff [5, Satz 5].

TheORem 2. Let the method $B$ be regular and let

$$
a_{\mu \nu}^{\prime} \rightarrow 0 \quad \text { as } \quad \mu \rightarrow \infty \text {. }
$$

Then

$$
[A, O(\Omega)] \subset[B, o(1) !
$$

if and only if

$$
\sum_{\nu=0}^{n}\left|\Omega(\nu) \sum_{\mu=\nu}^{n} b_{n \mu} a_{\mu \nu}^{\prime}\right|=o(1) .^{5}
$$

Proof. We write again

$$
\tau_{n}=\sum_{\mu=0}^{n} b_{n \mu} s_{\mu}
$$

In Theorem $2 \Omega(n)$ may be monotonic increasing or decreasing. 
then the inclusion (2) states that

$$
\sigma_{n}=O(\Omega(n)) \text { implies } \tau_{n}=o(1) .
$$

Using the inverse transformation we obtain

$$
\tau_{n}=\sum_{\mu=0}^{n} b_{n \mu} \sum_{\nu=0}^{\mu} a_{\mu \nu}^{\prime} \sigma_{\nu}=\sum_{\nu=0}^{n} \sigma_{\nu} \sum_{\mu=\nu}^{n} b_{n \mu} a_{\mu \nu}^{\prime} .
$$

Now we introduce the sequence $\left\{\delta_{\nu}\right\}$, where $\sigma_{\nu}=\Omega(\nu) \delta_{\nu}$ so that $\delta_{\nu}=O(1)$. Therefore

$$
\tau_{n}=\sum_{\nu=0}^{n} \delta_{\nu} \Omega(\nu) \sum_{\mu=\nu}^{n} b_{n \mu} a_{\mu \nu}^{\prime}=\sum_{\nu=0}^{n} c_{n \nu} \delta_{\nu},
$$

and $\left(c_{n v}\right)$ is a matrix method which transforms every bounded sequence $\left\{\delta_{\nu}\right\}$ into a null sequence $\left\{\tau_{n}\right\}$. Condition (1) implies that $c_{n \nu} \rightarrow 0$ as $n \rightarrow \infty$; therefore we can apply the theorem by Schur ${ }^{6}$ to the effect that under these circumstances

$$
\sum_{\nu=0}^{n}\left|c_{n \nu}\right|=o(1)
$$

as $n \rightarrow \infty$.

With this the first part of the theorem is proved. To show that equation (3) is sufficient for the inclusion (2) presents no difficulty.

REMARKs. (3) This theorem allows extensions similar to those mentioned in Remark 1.

(4) If the matrix $\left(a_{n v}\right)$ has the form

$$
\begin{array}{lll}
a_{n \nu}=1 & \text { for } & \nu \leqq n \\
a_{n \nu}=0 & \text { for } & \nu>n
\end{array}
$$

then $\Omega(n)$ is the "summability function of the second kind for the method $B$ " introduced by Lorentz [6].

TheOREM 3. Under the conditions of Theorem 2

$$
[A, O(\Omega)] \subset[B, O(1)] \quad([A, o(\Omega)] \subset[B, o(1)])
$$

if and only if

$$
\sum_{\nu=0}^{n}\left|\Omega(\nu) \sum_{\mu=\nu}^{n} b_{n \mu} a_{\mu \nu}^{\prime}\right|=O(1)
$$

The proof is similar to that of Theorem 2 .

3. Of the possible applications of these theorems we shall only

- See Schur [7]. 
mention a few. By taking the method $B$ in Theorem 2 to be the convergence matrix we obtain the

Corollary 1. Let $a_{n \nu}^{\prime} \rightarrow 0$ as $n \rightarrow \infty$, then

$$
\stackrel{A}{\sigma_{n}}=O(\Omega(n))
$$

is a Tauberian condition for the method $A$ if and only if

$$
\sum_{\nu=0}^{n}\left|\Omega(\nu) a_{n \nu}^{\prime}\right|=o(1)
$$

As a consequence of Theorem 3 we find as a known result. ${ }^{7}$

Corollary 2. A regular method $A$ is equivalent to convergence if and only if

$$
\sum_{\nu=0}^{n}\left|a_{n \nu}^{\prime}\right|=O(1)
$$

If, in Theorem 3 we take $A$ to be the method $\left(C, k^{\prime}\right)$ and $B$ to be the method $(C, k)$ where $k<k^{\prime}$, we find the

Corollary 3. Let the sequence $\left\{s_{n}\right\}$ be $\left(C, k^{\prime}\right)$-summable and let

$$
\sigma_{n}^{k^{\prime}}=O\left(n^{k-k^{\prime}}\right)
$$

then the sequence is $(C, k)$-summable.

Theorem 3 also furnishes a simple proof for the following wellknown theorem.

Theorem 4. Let the sequence $\left\{s_{n}\right\}$ be $(C, 1)$-summable and let

$$
\sigma_{n}=s+o\left(n^{-1 / 2}\right)
$$

then the sequence is Euler-summable. ${ }^{8}$

Proof. We take $A$ to be the $(C, 1)$-matrix and $B$ to be the Euler matrix. Then

$$
b_{n \mu}=\frac{1}{2^{n}}\left(\begin{array}{l}
n \\
\mu
\end{array}\right), \quad a_{\mu \nu}^{\prime} \begin{cases}=\nu & \text { for } \mu=\nu, \\
=-\nu & \text { for } \mu=\nu+1, \\
=0 & \text { otherwise, }\end{cases}
$$

and the left side of (4) becomes

7 See for instance Wilansky [8].

8 See for instance Hardy [2, p. 213]. 


$$
T_{n}=\sum_{\nu=1}^{n} \nu^{-1 / 2}\left|\nu \frac{1}{2^{n}}\left(\begin{array}{c}
n \\
\nu
\end{array}\right)-\nu \frac{1}{2^{n}}\left(\begin{array}{c}
n \\
\nu+1
\end{array}\right)\right|
$$

This can be rewritten in the form

$$
T_{n}=O(1) \sum_{\nu=1}^{n} \nu^{1 / 2}\left|\frac{1}{2^{n}}\left(\begin{array}{c}
n \\
\nu
\end{array}\right)-\frac{1}{2^{n}}\left(\begin{array}{c}
n \\
\nu+1
\end{array}\right)\right|,
$$

from which we obtain

$$
T_{n}=n^{1 / 2} \cdot \max _{o \leqq \nu \leqq n} \frac{1}{2^{n}}\left(\begin{array}{l}
n \\
\nu
\end{array}\right) O(1)=O(1) .
$$

Corollary 3 and Theorem 4 combine to

Corollary 4. Let the sequence $\left\{s_{n}\right\}$ be $(C, k)$-summable $(k>1)$ and let

$$
\stackrel{k}{\sigma_{n}}=s+o\left(n^{(1-2 k) / 2}\right),
$$

then the sequence is Euler-summable.

4. The investigations of the previous section can be extended to the case of absolute summability. One says that the sequence $\left\{s_{n}\right\}$ is absolutely $A$-summable, if

$$
\sum_{n=0}^{\infty}\left|\sigma_{n}-\sigma_{n+1}\right|<\infty .
$$

Also in use is the notation: $\sigma_{n}=a(\Omega(n))$ if

$$
\sum_{n=0}^{\infty}\left|\sigma_{n} \Omega^{-1}(n)-\sigma_{n+1} \Omega^{-1}(n+1)\right|<\infty .
$$

With this convention and using the notation of the proof of Theorem 2 we can state

TheOREM 5. The sequence $\left\{s_{n}\right\}$ is absolutely $B$-summable if

and if

$$
\sigma_{n}=a(\Omega(n))
$$

$$
\sum_{n=0}^{\infty} \sum_{\nu=0}^{n}\left|c_{n \nu}-c_{n+1, \nu}\right|<\infty .
$$

The proof is along the lines of the proof of Theorem 2. Application of this theorem shows, that Corollaries 3 and 4 still hold, if reworded for absolute summability. 


\title{
REFERENCES
}

1. L. S. Bosanquet, A mean value theorem, J. London Math. Soc. vol. 16 (1941) pp. 146-148.

2. G. H. Hardy, Divergent series, Oxford, 1949.

3. W. Jurkat, Über Rieszsche Mittel mit unstetigem Parameter, Math. Z. vol. 55 (1951) pp. 8-12.

4. IV. Jurkat and A. Peyerimhoff, Mittelwertsätze bei Matrix- und Integraltransformationen, Math. Z. vol. 55 (1951) pp. 92-108.

5. - Mittelwertsätze und Vergleichssätze für Matrixtransformationen, Math. Z. vol. 56 (1952) pp. 152-178.

6. G. G. Lorentz, Direct theorems on methods of summability, Canad. J. Math. vol. 1 (1949) pp. 305-319.

7. I. Schur, Über lineare Transformationen in der Theorie der unendlichen Reihen, J. Reine Angew. Math. vol. 151 (1921) pp. 79-111.

8. A. Wilansky, A necessary and sufficient condition that a summability method be stronger than convergence, Bull. Amer. Math. Soc. vol. 55 (1949) pp. 914-916.

University of New Mexico

\section{ON EXTENSIONS OF THE FIELD OF CONSTANTS OF AN ALGEBRAIC FUNCTION FIELD}

\author{
EVAR D. NERING
}

1. The purpose of this paper is to discuss the applications of a previous paper, Reduction of an algebraic function field modulo a prime in the constant ficld, [3], to cases where the constant field is extended. For completeness, we shall restate the principal results of that paper.

Let $K$ be an algebraic function field of one variable with constant field $k$, and let $k$ have a discrete non-Archimedean valuation $h$. Let $x$ be a fixed arbitrary element in $K$ transcendental over $k$. Let $h$ be extended to a valuation, also denoted by $h$, of $k(x)=F$ by the Gaussian definition. Then extend $h$ to valuations $H_{i}, i=1,2, \cdots, s$, on $K$. Let $K_{i}$ be the residue class field of $K$ modulo $H_{i}$. Let $\bar{k}$ and $\bar{F}$ denote the residue class fields of $k$ and $F$ modulo $h$. Properly interpreted, $K_{i}$ is finite algebraic over $\bar{k}(\bar{x})=\bar{F}$, i.e., $K_{i}$ is an algebraic function field. If $k_{i}$ is the constant field of $K_{i}$, then $k_{i}$ is of finite degree $r_{i}$ over $\bar{k}$. Let $g$ be the genus of $K$ and $g_{i}$ the genus of $K_{i}$. Let $e\left(H_{i}\right)$ be the ramification index of $H_{i}$. If the radical degree of each $H_{i}$ is 1 , then

Presented to the Society, August 28, 1957 under the title, An application of results on the reduction of an algebraic function field to extensions of the field of constants of an algebraic function field; received by the editors August 21, 1958. 